\title{
Comunicação
}

[Communication]

\section{Comparação entre as medidas morfométricas de equinos Mangalarga Marchador de marcha batida e marcha picada}

\author{
[Comparison between the morphometric measures of Mangalarga Marchador horses of batida \\ marcha and picada marcha] \\ J.M. Santiago, A.S.C. Rezende, Â.M.Q. Lana, M.G. Fonseca, R.G.P. Abrantes, J. Lage, \\ J.M. Andrade, T.M. Resende
}

Escola de Veterinária - Universidade Federal de Minas Gerais - Belo Horizonte, MG

Os equinos apresentam amplo espectro de funções devido às características anatômicas adquiridas em sua evolução. Sua variabilidade genética resulta em particularidades morfológicas e neurológicas, que conferem variadas formas de deslocamentos, caracterizando os diversos andamentos (Procópio et al., 2007).

O padrão da raça Mangalarga Marchador caracteriza a marcha como sendo um andamento natural, simétrico, a quatro tempos, com apoios alternados dos bípedes laterais e diagonais, intercalados por momentos de tríplice apoio, as reações são suaves, com pouco deslocamento vertical do centro de gravidade (Nascimento, 1999).

Procópio (2005) afirmou que existem grandes variações neste complexo andamento. Essas variações podem ser simplificadas com a definição da marcha picada, em que os membros se movimentam predominantemente com os bípedes laterais, aproximando-se, em seus extremos, da andadura. A marcha batida, por outro lado, apresenta predomínio dos deslocamentos dos bípedes em diagonal, aproximando-se do trote.

Ao utilizar análise cinemática, Procópio (2005) constatou que a marcha picada caracteriza-se por passadas de maior frequência e menor comprimento e que as proporções de apoios tripedais e laterais são superiores em relação à marcha batida. Considerando-se que a morfologia do equino é fundamental para a qualidade dos movimentos, inter-relacionando-se com a aptidão do animal (Nascimento, 1999), o presente estudo objetivou comparar as medidas morfométricas de equinos Mangalarga Marchador das categorias de marcha batida e marcha picada.

Este estudo foi aprovado pelo Comitê de Ética em Experimentação Animal da Universidade Federal de Minas Gerais (UFMG), sob o protocolo $n^{\circ} 152 / 2011$. A etapa experimental foi desenvolvida no Parque de Exposições Bolivar de Andrade, Belo Horizonte - MG, e o processamento dos dados foi realizado na Escola de Veterinária da UFMG.

Foram comparadas 22 medidas lineares e oito medidas angulares de 222 equinos machos (130 de marcha batida e 92 de marcha picada) e 266 equinos fêmeas (168 de marcha batida e 98 de marcha picada), todos credenciados anualmente com os títulos de campeão ou reservado campeão nas exposições regionais oficializadas pela Associação Brasileira de Criadores do Cavalo Mangalarga Marchador em todo o país e detentores dos títulos de campeão, reservado campeão, primeiro, segundo, terceiro, quarto ou quinto prêmio das categorias convencionais de marcha batida ou marcha picada, acima dos cinco anos, durante a $29^{\mathrm{a}}, 30^{\mathrm{a}}$ e $31^{\mathrm{a}}$ Exposição Nacional do Cavalo Mangalarga Marchador, realizadas em 2010, 2011 e 2012, respectivamente. 
O delineamento experimental foi inteiramente ao acaso, com dois tratamentos (marcha batida e marcha picada). As mensurações foram realizadas com os animais em estação forçada, isto é, membros, anterior e posterior, na perpendicular sobre um piso plano, formando um paralelogramo retangular, de forma que, vistos de perfil, seus membros, para cada bípede, encobrem-se e, vistos de frente ou por trás, estão na vertical e igualmente apoiados no solo. As medidas foram obtidas sempre do lado esquerdo do animal.

Os equipamentos utilizados nas mensurações foram um hipômetro, duas fitas métricas e um artrogoniômetro. Foram determinadas as medidas de altura na cernelha, garupa, dorso e costado, comprimento da cabeça, pescoço, espádua, braço, antebraço, canela e quartela do membro anterior, dorsolombo, garupa, coxa, perna, canela do membro posterior e corpo, largura da cabeça, peito e garupa, perímetro torácico e da canela do membro anterior, ângulo escapulossolo, escapuloumeral, umerorradial, metacarpofalangeano, pelvessolo, pelvefemoral, femorotibial e tibiotarsometatarsiano, de acordo com Zamborlini, et al. (1996), Lage et al. (2009) e Gonçalves et al. (2012).

As médias das medidas lineares foram submetidas ao Sistema Eclético de Proporções para o cavalo de sela (Lesbre, 1930), o qual determina que as medidas lineares sejam relacionadas ao comprimento da cabeça. Os dados foram testados quanto à distribuição normal e homocedasticidade entre os tratamentos. Os resultados foram submetidos à análise de variância, e as médias comparadas pelo teste de Fisher $(\mathrm{p}<0,05)$, utilizando-se o programa SAEG (versão 9.1).

Das 14 medidas lineares mensuradas na cabeça, pescoço e tronco dos equinos machos, houve diferenças $(\mathrm{P}<0,05)$ entre as categorias de marcha batida e marcha picada apenas no comprimento e largura da garupa e na largura do peito (Tab. 1). Já nos equinos fêmeas, houve diferença $(\mathrm{P}<0,05)$, entre as duas categorias de marcha, apenas no comprimento da garupa (Tab. 1).

As diferenças $(\mathrm{p}<0,05)$ encontradas entre as categorias de marcha batida e marcha picada, possivelmente, estão relacionadas com a movimentação que diferencia o andamento dos animais das duas categorias de marcha avaliadas. Essa hipótese é reforçada por Harris (1993), o qual afirmou que as medidas lineares e as angulações dos equinos determinam o tipo de movimento que eles são capazes de realizar. Por outro lado, a semelhança $(\mathrm{p}>0,05)$ de medidas, como comprimento da cabeça, comprimento do corpo e altura da cernelha, indica uniformidade dos equinos da raça Mangalarga Marchador, independentemente da categoria de marcha.

Tabela 1. Comparação entre medidas lineares de equinos Mangalarga Marchador (machos e femeas) das categorias de marcha batida e marcha picada

\begin{tabular}{lccccc}
\hline \multirow{2}{*}{ Medidas (cm) } & \multicolumn{2}{c}{ Machos } & & \multicolumn{2}{c}{ Fêmeas } \\
& Marcha batida & Marcha picada & & Marcha batida & Marcha picada \\
\cline { 1 - 2 } \cline { 5 - 6 } C. cabeça & $57,25 \pm 1,20$ & $57,46 \pm 1,50$ & & $57,39 \pm 1,68$ & $57,11 \pm 1,51$ \\
L. cabeça & $20,00 \pm 0,72$ & $19,93 \pm 0,66$ & & $19,63 \pm 0,66$ & $19,49 \pm 0,67$ \\
C. pescoço & $66,85 \pm 2,95$ & $67,05 \pm 2,80$ & & $65,76 \pm 3,11$ & $65,47 \pm 3,00$ \\
Alt. cernelha & $151,37 \pm 2,97$ & $150,88 \pm 2,63$ & & $149,92 \pm 3,15$ & $149,30 \pm 3,21$ \\
Alt. garupa & $149,12 \pm 3,00$ & $148,96 \pm 2,86$ & & $149,14 \pm 2,95$ & $148,32 \pm 3,15$ \\
C. corpo & $153,42 \pm 3,72$ & $152,67 \pm 3,81$ & & $155,17 \pm 3,57$ & $154,29 \pm 3,96$ \\
Alt. costado & $60,57 \pm 1,96$ & $61,05 \pm 1,93$ & & $61,89 \pm 1,86$ & $62,11 \pm 2,50$ \\
Alt. dorso & $141,14 \pm 2,73$ & $141,55 \pm 3,03$ & & $140,89 \pm 3,04$ & $140,52 \pm 3,01$ \\
Per. torácico & $173,17 \pm 4,42$ & $172,84 \pm 4,40$ & & $177,88 \pm 4,68$ & $176,34 \pm 5,34$ \\
C. dorsolombo & $48,27 \pm 2,42$ & $48,51 \pm 2,57$ & & $50,94 \pm 2,42$ & $51,80 \pm 2,72$ \\
C. garupa & $55,46 \mathrm{a} \pm 1,80$ & $54,74 \mathrm{~b} \pm 1,78$ & & $55,34 \mathrm{a} \pm 1,87$ & $54,60 \mathrm{~b} \pm 2,13$ \\
L. garupa & $50,45 \mathrm{a} \pm 1,51$ & $49,86 \mathrm{~b} \pm 1,91$ & & $52,76 \pm 1,95$ & $51,89 \pm 1,87$ \\
L. peito & $39,03 \mathrm{a} \pm 1,62$ & $38,31 \mathrm{~b} \pm 1,84$ & & $38,34 \pm 1,94$ & $37,43 \pm 1,78$ \\
\hline
\end{tabular}

Letras distintas nas linhas diferem entre as categorias marcha batida e marcha picada pelo teste de Fisher $(\mathrm{p}<0,05)$. Comprimento (C.), largura (L.), altura (Alt) e perímetro (Per.). 
O comprimento da garupa dos equinos machos e fêmeas da categoria de marcha batida foi superior ao de marcha picada. Segundo Nascimento (1999), uma garupa mais comprida possui músculos mais longos, resultando em maior capacidade de contração e amplitude das passadas dos membros posteriores. Procópio (2005) observou, em equinos de marcha batida, passadas de maior comprimento e menor frequência, em relação à marcha picada. Portanto, a garupa mais comprida dos equinos de marcha batida pode estar relacionada à necessidade de maior força de contração para obtenção de passadas mais amplas, quesito selecionado nos animais de marcha batida.

Embora os equinos machos de marcha batida tenham apresentado garupa mais larga e mais comprida em relação aos de marcha picada, a proporção entre o comprimento e a largura da garupa, que deve ser de 1:1 no cavalo de sela (Lesbre, 1930), foi inferior, tanto nos machos quanto nas fêmeas de ambas as categorias de marcha $(1: 0,9)$. Este resultado indica que tanto a garupa dos animais de marcha batida quanto a dos animais de marcha picada são proporcionalmente semelhantes, porém mais estreitas que o proposto no diagrama internacional do cavalo de sela. De acordo com Nascimento (1999), uma garupa larga pode prejudicar o deslocamento dos membros posteriores. Na raça Mangalarga Marchador, a seleção por animais com maior amplitude de movimentação dos membros posteriores e maior estabilidade do tronco, em especial da garupa, pode ter influenciado na menor proporção entre a largura e o comprimento de garupa verificada neste trabalho, em relação ao sistema de proporções do cavalo de sela (Lesbre,1930).

Os machos de marcha batida apresentaram peito mais largo que os de marcha picada, porém a proporção entre a largura do peito e o comprimento da cabeça de 0,68 e 0,67 , para as categorias de marcha batida e marcha picada, respectivamente, foi muito próxima. $\mathrm{O}$ peito muito largo é desejável no cavalo de tração, mas não no cavalo de sela, cujos andamentos são prejudicados pela exagerada separação dos membros anteriores (Nascimento, 1999).

Das nove medidas lineares mensuradas nos membros torácicos e pélvicos dos equinos machos, houve diferença $(\mathrm{P}<0,05)$ entre as duas categorias de marcha apenas no comprimento da quartela (Tab. 2), tendo os machos de marcha batida apresentado quartela mais comprida. O padrão da raça Mangalarga Marchador preconiza que a quartela tenha comprimento médio e, segundo Nascimento (1999), nos marchadores nacionais, quartelas compridas e inclinadas são desejáveis, pois se tornam mais flexíveis e dispersam mais facilmente as forças da locomoção, amortecendo bem as reações durante a locomoção.

Tabela 2. Médias e desvios-padrão das medidas lineares dos membros torácicos e pélvicos de equinos machos e fêmeas Mangalarga Marchador de marcha batida e marcha picada

\begin{tabular}{|c|c|c|c|c|}
\hline \multirow{2}{*}{ Medidas (cm) } & \multicolumn{2}{|c|}{ Machos } & \multicolumn{2}{|c|}{ Fêmeas } \\
\hline & Batida & Picada & Batida & Picada \\
\hline Comp. espádua & $50,73 \pm 1,52$ & $50,59 \pm 1,57$ & $50,70 a \pm 1,86$ & $49,92 b \pm 1,82$ \\
\hline Comp. braço & $29,89 \pm 1,45$ & $29,45 \pm 1,76$ & $29,04 \pm 2,54$ & $29,44 \pm 1,89$ \\
\hline Comp. antebraço & $41,40 \pm 1,54$ & $41,73 \pm 1,29$ & $41,11 b \pm 1,81$ & $41,79 a \pm 1,75$ \\
\hline Comp. canela ant. & $27,40 \pm 1,05$ & $27,22 \pm 1,30$ & $26,96 \pm 0,97$ & $26,75 \pm 0,96$ \\
\hline Perímetro canela & $19,29 \pm 0,65$ & $19,22 \pm 0,57$ & $10,68 \pm 0,93$ & $10,51 \pm 0,73$ \\
\hline Comp. quartela & $11,24 \mathrm{a} \pm 1,12$ & $10,77 b \pm 0,73$ & $18,60 \pm 0,62$ & $18,56 \pm 0,59$ \\
\hline Comp. coxa & $33,18 \pm 1,38$ & $33,30 \pm 1,67$ & $33,39 a \pm 1,75$ & $32,15 b \pm 1,98$ \\
\hline Comp. perna & $57,70 \pm 1,70$ & $58,01 \pm 1,90$ & $56,23 \pm 2,18$ & $57,41 \pm 2,23$ \\
\hline Comp. canela post. & $33,93 \pm 1,17$ & $34,10 \pm 1,28$ & $33,88 \pm 1,69$ & $33,91 \pm 1,63$ \\
\hline
\end{tabular}

Letras distintas nas linhas diferem entre as categorias marcha batida e marcha picada pelo teste de Fisher $(p<0,05)$. Comprimento (comp.), anterior (ant.) e posterior (post.).

As fêmeas de marcha batida apresentaram maior comprimento da espádua e coxa e menor comprimento do antebraço $(\mathrm{P}<0,05)$. Tanto o maior comprimento quanto a maior inclinação da espádua são importantes na qualidade de movimentação dos equinos, pois proporcionam maior amplitude da passada e favorecem o amortecimento do impacto do membro no solo, 
relacionando-se diretamente com a comodidade e o rendimento do andamento (Harris, 1993).

Lage et al. (2009), ao avaliarem as possíveis correlações entre as medidas morfométricas e os atributos da marcha de equinos Mangalarga Marchador, observaram associação positiva entre o comprimento da espádua e o rendimento, o que evidencia a importância dessa medida para a qualidade da marcha. Assim, o maior comprimento da espádua das fêmeas de marcha batida justifica-se pelo maior comprimento das passadas dos animais desta categoria de andamento (Procópio et al., 2007).O maior comprimento do antebraço facilita os movimentos de extensão e flexão dos complexos articulares e, assim, favorece a amplitude da passada. Nascimento (1999) relatou que o bom comprimento do antebraço é imprescindível nos cavalos de sela, devendo ser inversamente proporcional ao comprimento da canela, não prejudicar a extensão das passadas. Neste contexto, o antebraço mais comprido das fêmeas de marcha picada pode estar relacionado a uma compensação física, devido ao menor comprimento da espádua das fêmeas desta categoria, quando comparadas às éguas de marcha batida.

Em relação ao comprimento da coxa, o maior valor observado nas fêmeas de marcha batida é condizente com a maior amplitude da passada, o que foi observado por Procópio (2005), quando comparou a marcha batida com a marcha picada.

Houve diferença $(\mathrm{p}<0,05)$ nos ângulos escapulossolo e pelvessolo entre os equinos de marcha batida e marcha picada, machos e fêmeas. Mas, nos ângulos escapuloumeral e umerorradial, essa diferença $(\mathrm{p}<0,05)$ só ocorreu nos machos (Tab. 3).

Uma espádua bem inclinada permite ao membro anterior realizar movimentos mais avantes. Por outro lado, uma espádua mais vertical restringe a amplitude desse movimento e, consequentemente, diminui o comprimento da passada (Harris, 1993). Um braço mais verticalizado normalmente resulta em pequena elevação dos membros, mesmo quando associado a uma espádua mais oblíqua (Nascimento, 1999). Portanto, considerando-se que na marcha picada as passadas são mais curtas e que ocorre aumento da frequência destas para garantir manutenção da velocidade (Procópio, 2005), são coerentes os maiores ângulos articulares dos membros torácicos dos equinos de marcha picada, em relação à marcha batida, encontrados no presente trabalho. $\mathrm{O}$ menor ângulo umerorradial dos machos de marcha picada pode, mais uma vez, ser uma compensação física para as maiores angulação escapulossolo e escapuloumeral, favorecendo o amortecimento da passada e tornando o andamento mais cômodo.

Tabela 3. Médias, em graus, e desvios-padrão das medidas angulares de machos e fêmeas Mangalarga Marchador de marcha batida e marcha picada

\begin{tabular}{|c|c|c|c|c|}
\hline \multirow{2}{*}{ Medidas } & \multicolumn{2}{|c|}{ Machos } & \multicolumn{2}{|c|}{ Fêmeas } \\
\hline & Batida & Picada & Batida & Picada \\
\hline Â. escapulossolo & $58,94 \mathrm{~b} \pm 2,9$ & $61,20 \mathrm{a} \pm 3,9$ & $60,80 \mathrm{~b} \pm 3,2$ & $62,69 a \pm 3,4$ \\
\hline$\hat{A}$. escapuloumeral & $94,43 b \pm 4,4$ & $95,71 \mathrm{a} \pm 4,4$ & $95,82 \pm 4,4$ & $98,86 \pm 4,62$ \\
\hline$\hat{\text { A. }}$ umerorradial & $132,49 \mathrm{a} \pm 3,7$ & $131,3 b \pm 3,8$ & $133,70 \pm 3,9$ & $132,02 \pm 4,3$ \\
\hline Â. metacarpofalang. & $150,45 \pm 15,0$ & $149,1 \pm 4,6$ & $149,39 \pm 4,7$ & $150,03 \pm 4,5$ \\
\hline Â. pelvessolo & $28,48 b \pm 3,3$ & $30,08 \mathrm{a} \pm 3,1$ & $28,40 b \pm 9,4$ & $32,28 \mathrm{a} \pm 3,5$ \\
\hline$\hat{A}$. pelvefemoral & $82,24 \pm 4,8$ & $83,39 \pm 3,9$ & $86,81 \pm 5,1$ & $86,29 \pm 4,49$ \\
\hline Â. femorotibiopatelar & $109,60 \pm 5,0$ & $109,0 \pm 4,6$ & $111,14 \pm 4,8$ & $108,76 \pm 3,9$ \\
\hline Â. tibiotarsometatar & $141,43 \pm 5,1$ & $142,1 \pm 3,3$ & $142,42 \pm 3,8$ & $141,9 \pm 3,0$ \\
\hline Â. metatarsofalange & $145,61 \pm 4,7$ & $145,4 \pm 4,1$ & $146,19 \pm 4,2$ & $146,00 \pm 4,2$ \\
\hline
\end{tabular}

Letras distintas nas linhas diferem entre as categorias marcha batida e marcha picada pelo teste de Fisher $(\mathrm{p}<0,05)$.

Ângulo (Â.), falangeano (falang.) e metatarsiano (metatar.).

Os equinos machos e fêmeas de marcha picada apresentaram maior ângulo pelvessolo, o que indica uma garupa mais inclinada, porém ambas as categorias apresentaram ângulo pelvessolo dentro da faixa de 25 a 35 graus, considerada por
Thomas (2005) como representativo da garupa inclinada. Nascimento (1999) explicou que uma garupa mais horizontal é favorável à velocidade, com maior elevação do membro posterior, e que a garupa mais inclinada é favorável à força, 
sendo que o animal apresentará menor elevação dos membros posteriores, o que é compensado pelo aumento na frequência das passadas. Essa explicação justifica a maior angulação de garupa dos animais de marcha picada, os quais apresentam menor comprimento da passada, compensada pelo aumento de frequência dessas passadas durante sua dinâmica de movimentação.

Concluiu-se que a maioria das medidas lineares dos equinos Mangalarga Marchador de marcha batida e marcha picada apresentam valores semelhantes, indicando uniformidade do rebanho, independentemente da categoria de marcha. No entanto, esses animais apresentam diferenças nas medidas lineares e angulares relacionadas com a flexão dos membros, o comprimento e a frequência das passadas.

Palavras-chave: cavalo, medidas angulares, medidas lineares, locomoção

\begin{abstract}
The present study aimed to compare the morphometric measurements of Mangalarga Marchador horses of batida and picada marcha. Twenty-two linear and eight angular measurements of 222 males (130 of batida marcha and 92 of picada marcha) and 266 females (168 of batida marcha and 98 picada marcha) were compared in a completely randomized design, consisting of two treatments: horses of batida and picada marcha. The results were submitted to analysis of variance and means were compared by Fisher test $(P<0.05)$. It was concluded that most of the measures of Mangalarga Marchador horses of batida and picada marcha have similar values, however, there are differences between some angles of members.
\end{abstract}

Keywords: angular measurement, horse, locomotion, measure linear

\section{REFERÊNCIAS}

GONÇALVES, R.W.; COSTA, M.D.; REZENDE, A.S.C. et al. Efeito da endogamia sobre características morfométricas em cavalos da raça Mangalarga Marchador. Arq. Bras. Med. Vet. Zootec., v.64, p.419-426, 2012.

HARRIS, S.E. Horse gaits, balance and movement. New York: Howell Book House, 1993, 178p.

LAGE, M.C.G.R.; BERGMANN, J.A.G.; PROCÓPIO, A.M. et al. Associação entre medidas lineares e angulares de equinos da raça Mangalarga Marchador. Arq. Bras. Med. Vet. Zootec., v.61, p.968-979, 2009.

LESBRE, F.X. Precis d'Exterieur du Cheval. Paris. 1930.

NASCIMENTO, J.F. Mangalarga marchador: tratado morfofuncional. Belo Horizonte: ABCCMM, 1999. 577p.
PROCÓPIO, A.M. Análise cinemática da locomoção de equinos marchadores. 2005. 69f. Tese (Doutorado em Zootecnia) - Escola de Veterinária, Universidade Federal de Minas Gerais,Belo Horizonte.

PROCÓPIO, A.M.; BERGMANN, J.A.G.; MENZEL, H.J. et al. Curvas ângulo-tempo das articulações dos equinos marchadores. Arq. Bras. Med. Vet. Zootec., v.59, p.41-48, 2007.

THOMAS, H.S. The Horse Conformation. Storey Publishing, 2005. 387p.

ZAMBORLINI, L.C.; BERGMANN, J.A.G.; PEREIRA, C.S. et al. Estudo genéticoquantitativo de medidas lineares de equinos da raça mangalarga marchador - I. Estimativas dos fatores de ambiente e parâmetros genéticos. Rev. Bras. Cienc. Vet., v.3, p.33-37, 1996. 